\title{
Associations between diet and (in)activity behaviours with overweight and obesity among 10-18-year-old Czech Republic adolescents
}

\author{
Linda de Gouw ${ }^{1,2, *}+$, Knut-lnge Klepp ${ }^{2}$, Jana Vignerová ${ }^{3}$, Nanna Lien $^{2}$, \\ Ingrid HM Steenhuis ${ }^{1}$ and Marianne Wind ${ }^{2}$ \\ ${ }^{1}$ Faculty of Earth and Life Sciences, Department of Prevention and Public Health, Institute of Health Sciences, \\ VU University, De Boelelaan, Amsterdam, The Netherlands: ${ }^{2}$ Faculty of Medicine, Department of Nutrition, \\ University of Oslo, Boks 1072 Blindern, Oslo, Norway: ${ }^{3}$ Department of Health Promotion and Child and \\ Adolescent Health, National Institute of Public Health, Srobárova, Czech Republic
}

Submitted February 2009: Accepted May 2010

\begin{abstract}
Objectives: To assess the prevalence of normal weight, overweight and obesity among 10-18-year-old Czech Republic adolescents and to assess the association between energy balance-related behaviours and overweight/obesity.

Design: Cross-sectional data from 'The 6th Nation-wide Anthropological Survey of Children and Adolescents 2001 Czech Republic' have been used.

Setting: Height and weight were objectively measured. Data on adolescents' behaviours were assessed with self-reported questionnaires. Background variables were assessed by means of a parental questionnaire. Multi-level logistic regression analyses were conducted to assess behavioural risk factors of being overweight/obese.

Subjects: Data on 31228 adolescents aged 10-18 years were included in the present study.

Results: The highest prevalence of being overweight or obese was found among boys and younger adolescents. Boys were more physically active, watched more television (TV) and used the computer more often than did girls. Being on a diet and skipping meals were positively associated with being overweight/obese, independent of gender. In boys (10-14 years old), inverse associations with being overweight/obese were found when being more physically active. Monitoring weight showed inverse relations with being overweight/obese in 15-18-year-old girls. Watching TV more than $7 \mathrm{~h}$ a week was positively associated with being overweight/obese in 15-18-year-old girls, and was found to be negatively associated in boys of the same age group.

Conclusions: These behaviours should be targeted when preventing overweight and obesity among Czech Republic adolescents. Studies using better measures of energy balance-related behaviours are needed.
\end{abstract}

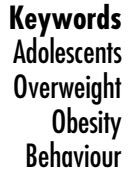

In the Czech Republic, not many data are available on the associations between behavioural and environmental factors and overweight or obesity ${ }^{(1,2)}$, although several studies from other countries have shown evidence of relations between these factors and overweight/obesity ${ }^{(3-6)}$.

In order to inform obesity prevention strategies among Czech Republic adolescents, there is a need for data on the prevalence of overweight and obesity and, equally important, insight into the relationship between energy balance-related behaviours and environmental factors on overweight and obesity in this country.

$\dagger$ Correspondence address: Enthovenplein 1, Postbus 1000, 2500 BA Den Haag, The Netherlands.
Therefore, the aim of the present study is 2-fold: (i) What was the prevalence of having a normal weight, being overweight and being obese (by using cut-off points of the International Obesity Task Force (IOTF) ${ }^{(7)}$ among 10-18-year-old Czech Republic adolescents? (ii) How were diet and (in)activity behaviours associated with being overweight/obese?

\section{Methods}

Cross-sectional data from 'The 6th Nation-wide Anthropological Survey of Children and Adolescents 2001 Czech

\section{Design and participants}


Republic' have been used ${ }^{(8)}$. A total of 281 schools from all over the country participated in this survey and were randomly recruited. Informed consent of parents was obtained before enrolment in the survey. Objective anthropometric measurements (height and weight) were collected by trained physical education teachers and pre-graduate students in Anthropology. Data on the behavioural aspects of adolescents were collected by means of questionnaires filled in by the adolescents under the guidance of their teacher during class hours. In addition, the adolescents brought a parent questionnaire home to be filled in by one or both of their parents ${ }^{(8)}$. Data of 31228 adolescents in the age group of 10-18 years were included in the present study.

\section{Measurements}

Based on the height and weight of adolescents, BMI was calculated using age- and gender-specific cut-off points from the $\operatorname{IOTF}^{(7)}$. The adolescents' questionnaire further contained questions on television (TV) viewing and use of the personal computer (PC) with answer possibilities ranging '0-3 h', ' $4-7 \mathrm{~h}$ ', '8-14 h' and 'more than $14 \mathrm{~h}$ ' a week. TV viewing was later dichotomized into 'up to $7 \mathrm{~h} \mathrm{a}$ week' and 'more than $7 \mathrm{~h}$ a week', while PC use was dichotomized into 'up to 3 h' and 'more than 3 h' a week. The amount of physical activity was assessed by estimating the amount of hours being physically active in a week and was later dichotomized as 'up to $7 \mathrm{~h}$ a week' and 'more than $7 \mathrm{~h}$ a week'. Daily consumption of meals was assessed with questions on breakfast, mid-morning snack, lunch, afternoon snack and something besides these meals. These questions could be answered by 'yes' or 'no'. In addition, being on a diet ('yes'/no') and monitoring weight ('yes'/'no') were also assessed.

The parents' questionnaires contained questions on self-reported height and weight of both the father and the mother, education of both parents (elementary school, apprenticeship, secondary school and university graduate), size of the residence they lived in (up to 5000, up to 10000 , up to 100000 , up to 1000000 and more than 1000000 inhabitants), birth weight and height of the child, whether the child had been breast-fed (not breastfed, up to 1 month, up to 3 months, up to 6 months and more than 6 months), number of children in the family and if the child was pre-pubescent or pubescent ('before voice breaking' or 'after voice breaking' and for girls 'before menarche' or 'after menarche').

Based on their self-reported height and weight, BMI values of both parents were calculated. Normal weight was defined as BMI $<25 \mathrm{~kg} / \mathrm{m}^{2}$, overweight as BMI $\geq 25 \mathrm{~kg} / \mathrm{m}^{2}$ and obese as BMI $\geq 30 \mathrm{~kg} / \mathrm{m}^{2}$. Parental BMI was then divided into three groups ('both parents normal weight', 'one parent overweight or obese' and 'both parents overweight or obese'). Education of the parents was also divided into three groups ('both parents lower education', 'one parent higher education' and 'both parents higher education'). Lower education contained 'elementary school', 'elementary school' and 'apprenticeship' and higher education contained 'secondary school', 'secondary school' and 'university graduation'.

Size of residence was dichotomized in 'up to 10000 inhabitants' and 'more than 10000 inhabitants'. Breastfeeding was subdivided into three categories (not breastfed, up to 3 months and more than 3 months). Finally, the number of children in the family was dichotomized as 'up to two children in the family' and 'more than two children in the family'.

\section{Statistical analysis}

First, $\chi^{2}$ and univariate ANOVA tests were used to assess differences in energy balance-related behaviours and background variables among the three weight groups (normal weight, overweight and obese) and to test differences between both genders within each weight group. Each factor was analysed separately among the three weight groups and between genders within weight groups. Second, Spearman-Pearson correlations among all variables were computed to assess unadjusted correlations between independent variables, to check for possible multi-collinearity, and between independent variables and BMI. An intercorrelation of 0.50 was allowed as a maximum correlation between two independent variables ${ }^{(9)}$.

To assess associations between behaviours and being overweight/obese, stepwise multi-level logistic regression models were run on dichotomized BMI (normal weight $v$. overweight/obese), accounting for the clustering of individuals within schools.

First, each behavioural factor (i.e. physical activity, TV viewing, PC use, breakfast, lunch, mid-morning snack, afternoon snack, besides meals snack, monitoring weight and dieting) was separately regressed on the outcome measure. The second model contained all the behavioural factors, and birth weight, puberty, BMI of the parents, education of the parents, number of children in the family, breast-feeding and the number of inhabitants the child lives with since previous studies have shown that these factors are related to $\mathrm{BMI}^{(6,10)}$. Both models were adjusted for age and school, and were tested separately for boys and girls and two age groups: $10-14$ and 15-18 years. Statistical analyses were performed using the Statistical Package for Social Sciences statistical software package version $15 \cdot 0 \cdot 1$ (SPSS Inc., Chicago, IL, USA) and MLwiN version $2 \cdot 12^{(11)}$. A statistical significance level of $P<0 \cdot 01$ was used for differences in all independent variables within the three body weight groups and between both genders. For multi-level logistic regression models, $P<0.05$ (two-sided) was considered statistically significant.

\section{Non-respondents}

Adolescents were excluded from logistic regression models when having missing data on any of the energy-balanced behavioural factors or background factors ( $n$ 19716). 
Logistic regression analysis was performed to check for differences in age, gender and BMI between included and excluded adolescents.

\section{Results}

\section{Characteristics of the study population}

Table 1 shows the prevalence of having a normal weight, being overweight and being obese in boys and girls of all ages. Statistically significant differences between boys and girls on BMI were found, with more boys being overweight or obese than girls $(P<0 \cdot 01)$. Lower prevalence of overweight and obesity was found when age was higher.

Dropout analysis showed that the adolescents who were excluded from the logistic regression analysis were more often boys (OR $=0 \cdot 91,95 \%$ CI $0 \cdot 87,0 \cdot 96)$, older $(\mathrm{OR}=1 \cdot 02,95 \% \mathrm{CI} 1 \cdot 01,1 \cdot 03)$ and overweight $(\mathrm{OR}=$ $1 \cdot 26,95 \% \mathrm{CI} 1 \cdot 16,1 \cdot 36)$ or obese (OR $=1 \cdot 64,95 \% \mathrm{CI}$ $1 \cdot 36,1 \cdot 98)$ compared to the adolescents who were included in the analysis (data not shown).

As Table 2 shows, 'obese' adolescents were more likely to be younger $(P<0 \cdot 001)$ and less likely to be breast-fed for more than 3 months $(P<0 \cdot 001)$, had more often parents who were both overweight/obese $(P<0 \cdot 001)$ or who both completed lower education $(P<0 \cdot 001)$, were more likely to come from small residences (up to 10000 inhabitants; $P<0 \cdot 001)$, skipped a meal more often $(P<0 \cdot 001)$ and were more often on a diet $(P<0 \cdot 001)$ than their overweight or normal weight peers.

Table 1 Prevalence of BMI, categorized in normal weight, overweight and obesity based on age- and gender-specific cut-off points from the IOTF for 10-18-year-old adolescents from 'The 6th Nation-wide Anthropological Survey of Children and Adolescents 2001 Czech Republic'

\begin{tabular}{|c|c|c|c|c|c|c|c|}
\hline & \multirow[b]{2}{*}{ Total $(n)$} & \multicolumn{2}{|c|}{ Normal weight } & \multicolumn{2}{|c|}{ Overweight } & \multicolumn{2}{|c|}{ Obese } \\
\hline & & $n$ & $\%$ & $n$ & $\%$ & $n$ & $\%$ \\
\hline \multicolumn{8}{|c|}{ Boys (Age, years) } \\
\hline 10 & 1397 & 1128 & $80 \cdot 7$ & 217 & $15 \cdot 5$ & 52 & $3 \cdot 7$ \\
\hline 11 & 1495 & 1213 & $81 \cdot 1$ & 238 & $15 \cdot 9$ & 44 & $2 \cdot 9$ \\
\hline 12 & 1679 & 1356 & $80 \cdot 8$ & 280 & $16 \cdot 7$ & 43 & $2 \cdot 6$ \\
\hline 13 & 1693 & 1426 & $84 \cdot 2$ & 224 & $13 \cdot 2$ & 43 & $2 \cdot 5$ \\
\hline 14 & 1449 & 1248 & $86 \cdot 1$ & 167 & $11 \cdot 5$ & 34 & $2 \cdot 3$ \\
\hline 15 & 1643 & 1429 & $87 \cdot 0$ & 186 & $11 \cdot 3$ & 28 & $1 \cdot 7$ \\
\hline 16 & 1831 & 1625 & $88 \cdot 7$ & 169 & $9 \cdot 2$ & 37 & $2 \cdot 0$ \\
\hline 17 & 1498 & 1335 & $89 \cdot 1$ & 142 & $9 \cdot 5$ & 21 & $1 \cdot 4$ \\
\hline 18 & 1015 & 874 & $86 \cdot 1$ & 117 & $11 \cdot 5$ & 24 & $2 \cdot 4$ \\
\hline \multicolumn{8}{|c|}{ Girls (Age, years) } \\
\hline 10 & 1465 & 1209 & $82 \cdot 5$ & 218 & $14 \cdot 9$ & 38 & $2 \cdot 6$ \\
\hline 11 & 1644 & 1386 & $84 \cdot 3$ & 216 & $13 \cdot 1$ & 42 & $2 \cdot 6$ \\
\hline 12 & 1636 & 1393 & $85 \cdot 1$ & 217 & $13 \cdot 3$ & 26 & $1 \cdot 6$ \\
\hline 13 & 1578 & 1393 & $88 \cdot 3$ & 163 & $10 \cdot 3$ & 22 & $1 \cdot 4$ \\
\hline 14 & 1491 & 1340 & $89 \cdot 9$ & 135 & $9 \cdot 1$ & 16 & $1 \cdot 1$ \\
\hline 15 & 2544 & 2333 & $91 \cdot 7$ & 188 & $7 \cdot 4$ & 23 & 0.9 \\
\hline 16 & 2678 & 2457 & $91 \cdot 7$ & 200 & $7 \cdot 5$ & 21 & 0.8 \\
\hline 17 & 2533 & 2340 & $92 \cdot 4$ & 175 & $6 \cdot 9$ & 18 & 0.7 \\
\hline 18 & 1697 & 1569 & 92.5 & 104 & $6 \cdot 1$ & 24 & 1.4 \\
\hline
\end{tabular}

IOTF $=$ International Obesity Task Force.

Percentages were count within rows.
Overweight adolescents were more likely to come from small residences $(P<0 \cdot 001)$ than normal-weight adolescents. Overweight adolescents skipped meals more often and were on a diet more often than normal-weight adolescents, but less often than obese adolescents (both $P<0 \cdot 001)$.

Normal-weight adolescents were older $(P<0 \cdot 001)$, more likely to live in large residences $(P<0 \cdot 001)$ and less likely to be pre-pubescent $(P<0 \cdot 001)$, more often had parents who both completed higher education $(P<0 \cdot 001)$, were more often $>7 \mathrm{~h}$ physically active in a week $(P<0 \cdot 01)$, were more likely to spend $>3 \mathrm{~h}$ a week behind the computer than overweight or obese adolescents $(P<0 \cdot 01)$ and less likely to skip a meal $(P<0 \cdot 001)$ or be on a diet $(P<0 \cdot 001)$ in comparison with overweight or obese adolescents.

Gender differences were found within the three weight groups. Normal weight and overweight boys were more likely to be pre-pubescent (both $P<0 \cdot 001$ ) and have parents who had both completed higher education (both $P<0.001)$ than normal-weight and overweight girls. In every weight group, boys were more physically active $(P<0 \cdot 001)$, used the PC more often $(P<0 \cdot 001)$ and watched more TV $(P<0 \cdot 001)$ than girls, while more girls than boys were on a diet $(P<0 \cdot 01)$. Normal-weight and overweight girls more often skipped a meal $(P<0 \cdot 01)$ and were more likely to monitor their weight than normalweight and overweight boys $(P<0 \cdot 001)$. Intercorrelations among all independent variables were below 0.50 and most variables were significantly correlated to BMI.

\section{Associations between energy balance-related behaviours and being overweight or obese}

All multi-level logistic regression analyses were first analysed naïve, without school. Adding school as a clustering variable to the model hardly changed the odds ratios. Further, adding behavioural variables to the model led to an overall decreased number of factors that were statistically significantly associated with being overweight or obese. Therefore, only adjusted odds ratios are shown in Table 3 and results of the final models (step 2, Table 3) are described below.

Among boys in both age groups, the risk for being overweight/obese was associated with not having consumed an afternoon snack (10-14-year-olds: OR $=1 \cdot 43$, 95\% CI $1 \cdot 11,1 \cdot 84,15-18$-year-olds: OR $=1 \cdot 56,95 \% \mathrm{CI}$ $1 \cdot 14,2 \cdot 15)$ and skipping a besides meal snack (10-14year-olds: $\mathrm{OR}=1 \cdot 41,95 \% \mathrm{CI} 1 \cdot 12,1 \cdot 77,15-18$-year-olds: $\mathrm{OR}=1 \cdot 71,95 \%$ CI $1 \cdot 27,2 \cdot 28)$. Slightly higher odds were found in 15-18-year-old boys than in 10-14-year-old boys. Dieting (OR $=4 \cdot 60,95 \%$ CI 3·12, 6.78) was positively associated with overweight/obesity in 10-14-yearold boys. Among the older boys, dieting was no longer significantly associated with being overweight/obese, after adjusting for energy balance-related behaviours and background factors. Being physically active for $>7 \mathrm{~h} /$ week $(\mathrm{OR}=0.74,95 \% \mathrm{CI} 0 \cdot 59,0.93)$ showed an 
Table 2 Characteristics of 10-18-year-old Czech Republic adolescents from 'The 6th Nation-wide Anthropological Survey of Adolescents 2001 Czech Republic' on BMI, based on age- and gender-specific cut-off points from the IOTF, corrected for gender

\begin{tabular}{|c|c|c|c|c|c|c|c|c|c|c|c|c|c|}
\hline \multirow[b]{3}{*}{ Sex } & \multirow[b]{3}{*}{$n$} & \multicolumn{4}{|c|}{ Normal weight } & \multicolumn{4}{|c|}{ Overweight } & \multicolumn{4}{|c|}{ Obese } \\
\hline & & \multicolumn{2}{|c|}{ Boys } & \multicolumn{2}{|l|}{ Girls } & \multicolumn{2}{|l|}{ Boys } & \multicolumn{2}{|l|}{ Girls } & \multicolumn{2}{|l|}{ Boys } & \multicolumn{2}{|l|}{ Girls } \\
\hline & & Mean or $\%$ & SD & Mean or \% & SD & Mean or \% & SD & Mean or $\%$ & SD & Mean or $\%$ & SD & Mean or $\%$ & SD \\
\hline Age & 30966 & $14 \cdot 5$ & $2 \cdot 5^{*} \dagger$ & $15 \cdot 0$ & $2 \cdot 5$ & $13 \cdot 9$ & $2 \cdot 5$ & $14 \cdot 1$ & $2 \cdot 6$ & $13 \cdot 8$ & $2 \cdot 5$ & $13 \cdot 9$ & $2 \cdot 7$ \\
\hline BMI $\left(\mathrm{kg} / \mathrm{m}^{2}\right)$ & 30966 & $19 \cdot 1$ & $2 \cdot 3^{*} \dagger$ & $19 \cdot 4$ & $2 \cdot 3$ & $24 \cdot 0$ & $2 \cdot 0+$ & $24 \cdot 5$ & $2 \cdot 1$ & $29 \cdot 2$ & $2 \cdot 8$ & $29 \cdot 7$ & $3 \cdot 1$ \\
\hline Birth weight & 18471 & $3 \cdot 4$ & $0.5^{*} \dagger$ & $3 \cdot 3$ & & $3 \cdot 5$ & $0.5+$ & $3 \cdot 3$ & 0.5 & 3.5 & 0.5 & $3 \cdot 3$ & $0 \cdot 4$ \\
\hline Breast-feeding (\%)* & 18204 & \multicolumn{4}{|c|}{ Breast-feeding ${ }^{*}$} & \multicolumn{4}{|c|}{ Breast-feeding } & \multicolumn{4}{|c|}{ Breast-feeding } \\
\hline Not breast-fed & & $6 \cdot 8$ & & 6.9 & & $8 \cdot 3$ & & $9 \cdot 6$ & & $10 \cdot 2$ & & $11 \cdot 2$ & \\
\hline Up to 3 months & & $51 \cdot 1$ & & $51 \cdot 9$ & & $49 \cdot 2$ & & $48 \cdot 5$ & & $56 \cdot 3$ & & $58 \cdot 6$ & \\
\hline More than 3 months & & $42 \cdot 1$ & & $41 \cdot 2$ & & $42 \cdot 5$ & & 41.9 & & 33.5 & & $30 \cdot 2$ & \\
\hline Puberty (\%) & 18173 & & Puber & $\operatorname{ty}^{*}+$ & & & Pube & ertyt & & & Pube & erty & \\
\hline Before voice breaking/menarche & & $41 \cdot 6$ & & $25 \cdot 6$ & & $50 \cdot 4$ & & $32 \cdot 5$ & & $49 \cdot 4$ & & $39 \cdot 0$ & \\
\hline After voice breaking/menarche & & $58 \cdot 4$ & & $74 \cdot 4$ & & $49 \cdot 6$ & & $67 \cdot 5$ & & $50 \cdot 6$ & & $61 \cdot 0$ & \\
\hline Parents' BMI (kg/m²; \%) & 21068 & & arents' & $\mathrm{BMI}^{\star}+$ & & & arents & s' BMIt & & & rents & 's' BMI & \\
\hline Both parents normal weight & & $29 \cdot 0$ & & $27 \cdot 5$ & & $15 \cdot 6$ & & $14 \cdot 2$ & & $26 \cdot 9$ & & $5 \cdot 8$ & \\
\hline One parent overweight/obese & & $52 \cdot 0$ & & $51 \cdot 6$ & & $53 \cdot 7$ & & $48 \cdot 1$ & & $39 \cdot 8$ & & $42 \cdot 4$ & \\
\hline Both parents overweight/obese & & $19 \cdot 0$ & & $20 \cdot 8$ & & $30 \cdot 7$ & & $37 \cdot 7$ & & $53 \cdot 4$ & & $51 \cdot 8$ & \\
\hline Parents' education (\%) & 21765 & Pare & ints' ed & lucation ${ }^{*}+$ & & Pare & nts' ec & ducationt & & Pare & nts' $e$ & education & \\
\hline Both parents lower educated & & $23 \cdot 1$ & & $24 \cdot 9$ & & $26 \cdot 7$ & & $34 \cdot 0$ & & $36 \cdot 7$ & & $38 \cdot 8$ & \\
\hline One parent higher educated & & $29 \cdot 3$ & & $31 \cdot 1$ & & $30 \cdot 1$ & & $31 \cdot 5$ & & $34 \cdot 2$ & & $34 \cdot 7$ & \\
\hline Both parents higher educated & & $47 \cdot 6$ & & $44 \cdot 0$ & & $43 \cdot 3$ & & $34 \cdot 2$ & & $29 \cdot 1$ & & $26 \cdot 5$ & \\
\hline Residence size (\%) & 28212 & & esidenc & size ${ }^{*}$ & & & siden & ce size & & $\operatorname{Res}$ & siden & ce size & \\
\hline Up to 10000 inhabitants & & $52 \cdot 2$ & & $52 \cdot 2$ & & $55 \cdot 4$ & & $56 \cdot 5$ & & $61 \cdot 2$ & & $59 \cdot 7$ & \\
\hline$>10000$ inhabitants & & $47 \cdot 8$ & & $47 \cdot 8$ & & $44 \cdot 6$ & & $43 \cdot 5$ & & $38 \cdot 8$ & & $40 \cdot 3$ & \\
\hline Family size (\%) & 18553 & & Family & sizet & & & Family & y size & & & amily & y size & \\
\hline 1 or 2 children & & $74 \cdot 3$ & & $71 \cdot 3$ & & NS & & NS & & NS & & NS & \\
\hline$>2$ children & & $25 \cdot 7$ & & $28 \cdot 7$ & & NS & & NS & & NS & & NS & \\
\hline Physical activity (\%) & 18464 & & ysical a & activity + & & $\mathrm{Phy}$ & sical & activityt & & Phy & sical & activity ${ }^{+}$ & \\
\hline Up to $7 \mathrm{~h} /$ week & & $37 \cdot 2$ & & $61 \cdot 2$ & & $41 \cdot 0$ & & $62 \cdot 6$ & & 41.9 & & $64 \cdot 9$ & \\
\hline$>7 \mathrm{~h} /$ week & & $62 \cdot 8$ & & $38 \cdot 8$ & & $59 \cdot 0$ & & $37 \cdot 4$ & & $58 \cdot 1$ & & $35 \cdot 1$ & \\
\hline TV watching (\%) & 19417 & & $V$ watc & $h_{i n g}^{*}+$ & & & $\checkmark$ wat & tching $\dagger$ & & & wat & tching $\dagger^{-}$ & \\
\hline Up to $7 \mathrm{~h} /$ week & & $43 \cdot 0$ & & $57 \cdot 9$ & & $38 \cdot 3$ & & $51 \cdot 8$ & & $38 \cdot 3$ & & 56.9 & \\
\hline$>7 \mathrm{~h} /$ week & & $57 \cdot 0$ & & $42 \cdot 1$ & & $56 \cdot 8$ & & $48 \cdot 2$ & & $61 \cdot 7$ & & $43 \cdot 1$ & \\
\hline PC use (\%) & 19140 & & PC us & $\mathrm{se}^{\star} t$ & & & $\mathrm{PC} \mathrm{u}$ & uset & & & PC u & uset & \\
\hline Up to $3 \mathrm{~h} /$ week & & $37 \cdot 7$ & & $74 \cdot 0$ & & $39 \cdot 4$ & & 74.5 & & $39 \cdot 4$ & & 73.9 & \\
\hline$>3 \mathrm{~h} /$ week & & $62 \cdot 3$ & & $26 \cdot 0$ & & $60 \cdot 2$ & & $25 \cdot 5$ & & $60 \cdot 6$ & & $26 \cdot 1$ & \\
\hline Breakfast (\%) & 19606 & & Breakf & $\operatorname{ast}^{*}+$ & & & Break & kfastt & & & Break & kfast & \\
\hline Yes & & $74 \cdot 0$ & & $64 \cdot 3$ & & $67 \cdot 4$ & & $60 \cdot 3$ & & $60 \cdot 7$ & & 52.5 & \\
\hline No & & $26 \cdot 0$ & & $35 \cdot 7$ & & $32 \cdot 6$ & & $39 \cdot 7$ & & $39 \cdot 3$ & & $47 \cdot 5$ & \\
\hline Lunch (\%) & 19546 & & Luncl & & & & Lunc & & & & Lun & & \\
\hline Yes & & $77 \cdot 0$ & & $69 \cdot 1$ & & $71 \cdot 1$ & & $61 \cdot 5$ & & $63 \cdot 0$ & & $63 \cdot 6$ & \\
\hline No & & $23 \cdot 0$ & & $30 \cdot 9$ & & $28 \cdot 9$ & & $38 \cdot 5$ & & $37 \cdot 0$ & & $36 \cdot 4$ & \\
\hline Mid-morning snack (\%) & 19551 & Mid- & morning & g snack ${ }^{*} \dagger$ & & Mid- & mornir & ing snack & & Mid-n & norni & ing snack & \\
\hline Yes & & $88 \cdot 8$ & & $86 \cdot 5$ & & $85 \cdot 9$ & & $84 \cdot 7$ & & $76 \cdot 8$ & & $82 \cdot 1$ & \\
\hline No & & $11 \cdot 2$ & & 13.5 & & $14 \cdot 1$ & & $15 \cdot 3$ & & $23 \cdot 2$ & & $17 \cdot 9$ & \\
\hline Afternoon snack (\%) & 19418 & & ernoon & snack $^{*} \dagger$ & & Afte & rnoon & n snackt & & Afte & rnoo & n snack & \\
\hline Yes & & $77 \cdot 3$ & & $74 \cdot 4$ & & $70 \cdot 1$ & & $64 \cdot 3$ & & $61 \cdot 8$ & & $58 \cdot 0$ & \\
\hline No & & $22 \cdot 7$ & & $25 \cdot 6$ & & $29 \cdot 9$ & & $35 \cdot 7$ & & $38 \cdot 2$ & & $42 \cdot 0$ & \\
\hline Besides meals snack (\%) & 19429 & Besid & es mea & als snack ${ }^{*}+$ & & Besid & me & als snackt & & Beside & $\mathrm{se}$ & eals snack & \\
\hline Yes & & $67 \cdot 5$ & & 56.9 & & $58 \cdot 2$ & & $50 \cdot 6$ & & $49 \cdot 4$ & & $47 \cdot 5$ & \\
\hline No & & $32 \cdot 5$ & & $43 \cdot 1$ & & $41 \cdot 8$ & & $49 \cdot 4$ & & $50 \cdot 6$ & & $52 \cdot 5$ & \\
\hline Monitoring weight (\%) & 19547 & Mo & nitoring & weightt & & Mon & itoring & g weightt & & Mon & torin & g weight & \\
\hline Yes & & $45 \cdot 5$ & & $68 \cdot 8$ & & $52 \cdot 6$ & & $67 \cdot 3$ & & $56 \cdot 1$ & & $64 \cdot 7$ & \\
\hline No & & $54 \cdot 5$ & & $31 \cdot 5$ & & $47 \cdot 4$ & & $32 \cdot 7$ & & $43 \cdot 9$ & & $35 \cdot 3$ & \\
\hline Dieting (\%) & 19516 & & Dietin & $\lg ^{*}+$ & & & Dieti & ingt & & & Dieti & ingt & \\
\hline Yes & & $2 \cdot 9$ & & $11 \cdot 0$ & & $10 \cdot 2$ & & $26 \cdot 2$ & & $21 \cdot 4$ & & $38 \cdot 7$ & \\
\hline No & & $97 \cdot 1$ & & $89 \cdot 0$ & & $89 \cdot 8$ & & $73 \cdot 8$ & & $78 \cdot 6$ & & $61 \cdot 3$ & \\
\hline
\end{tabular}

IOTF, International Obesity Task Force.

Percentages were counted by categorized BMI and within sex.

${ }^{*}$ Statistical significance of $<0.01$ on categorized BMI, performed with one-way ANOVA or $\chi^{2}$ test.

tStatistical significance of $<0.01$ between sex in each BMI group, performed with one-way ANOVA or $\chi^{2}$ test.

inverse relationship with overweight/obesity in 10-14year-old boys. Skipping breakfast $(\mathrm{OR}=1 \cdot 33,95 \% \mathrm{CI}$ $1 \cdot 04,1 \cdot 70)$ was associated with an increased risk for being overweight/obese in 10-14-year-old boys, but no longer among 15-18-year-old boys after adding behavioural factors and background factors in the model (step 2).
Skipping lunch $(\mathrm{OR}=1 \cdot 65,95 \% \mathrm{CI} 1 \cdot 18,2 \cdot 30)$ and skipping a mid-morning snack (OR $=1 \cdot 76,95 \%$ CI $1 \cdot 25$, $2 \cdot 50$ ) were positively related to overweight/obesity in 15-18-year-old boys, and watching TV for $>7 \mathrm{~h} /$ week $(\mathrm{OR}=0.69,95 \% \mathrm{CI} 0 \cdot 51,0.92)$ showed an inverse relation with overweight/obesity. 
Table 3 Stepwise multi-level logistic regression model of 10-14 and 15-18-year-old boys and girls from 'The 6th Nation-wide Anthropological Survey of Children and Adolescents 2001 Czech Republic' with overweight/obesity as a dependent variable, based on age- and gender-specific cut-off points from the IOTF

\begin{tabular}{|c|c|c|c|c|c|c|c|c|}
\hline & \multicolumn{4}{|c|}{ Boys } & \multicolumn{4}{|c|}{ Girls } \\
\hline & \multicolumn{2}{|c|}{ Step 1‡ } & \multicolumn{2}{|c|}{ Step $2 \S$} & \multicolumn{2}{|c|}{ Step 1‡ } & \multicolumn{2}{|c|}{ Step $2 \S$} \\
\hline & OR & $95 \% \mathrm{Cl}$ & OR & $95 \% \mathrm{Cl}$ & OR & $95 \% \mathrm{Cl}$ & OR & $95 \% \mathrm{Cl}$ \\
\hline $10-14$ years old & \multicolumn{2}{|c|}{$(n 2711)$} & \multicolumn{2}{|c|}{$(n 2711)$} & \multicolumn{2}{|c|}{ (n 2909) } & \multicolumn{2}{|c|}{ (n 2909) } \\
\hline Physical activity & & & & & & & & \\
\hline $\begin{array}{l}\text { Up to } 7 \mathrm{~h} / \text { week } \\
>7 \mathrm{~h} / \text { week }\end{array}$ & $\begin{array}{l}1 \cdot 00 \\
0 \cdot 76\end{array}$ & $\begin{array}{c}\text { Ref. } \\
\mathbf{0 . 6 2 , 0 . 9 5}\end{array}$ & 0.74 & $0.59,0.93$ & $\begin{array}{l}1.00 \\
\mathbf{0} \cdot 79\end{array}$ & $\begin{array}{c}\text { Ref. } \\
0.63,0.99\end{array}$ & $\begin{array}{l}1.00 \\
0.82\end{array}$ & $\begin{array}{c}\text { Ref. } \\
0.66,1.05\end{array}$ \\
\hline \multicolumn{9}{|l|}{ TV watching } \\
\hline Up to $7 \mathrm{~h} /$ week & $1 \cdot 00$ & Ref. & 1.00 & Ref. & 1.00 & Ref. & 1.00 & Ref. \\
\hline$>7 \mathrm{~h} /$ week & $1 \cdot 13$ & $0.91,1.41$ & $1 \cdot 09$ & $0.86,1 \cdot 37$ & $1 \cdot 14$ & $0.91,1.42$ & $1 \cdot 14$ & $0.89,1.45$ \\
\hline \multicolumn{9}{|l|}{ PC use } \\
\hline Up to $3 \mathrm{~h} /$ week & 1.00 & Ref. & 1.00 & Ref. & 1.00 & & 1.00 & Ref. \\
\hline$>3 \mathrm{~h} /$ week & $1 \cdot 03$ & $0.83,1.28$ & 0.98 & $0 \cdot 78,1 \cdot 24$ & 0.90 & $0 \cdot 70,1 \cdot 16$ & 0.83 & $0.63,1.09$ \\
\hline Breakfastll & $1 \cdot 48$ & $1 \cdot 17,1 \cdot 87$ & $1 \cdot 33$ & $1 \cdot 04,1 \cdot 70$ & $1 \cdot 45$ & $1 \cdot 16,1 \cdot 83$ & $1 \cdot 24$ & $0.97,1.58$ \\
\hline Lunchll & $1 \cdot 13$ & $0 \cdot 87,1.45$ & 0.98 & $0.74,1 \cdot 30$ & $1 \cdot 30$ & $1 \cdot 00,1 \cdot 71$ & $1 \cdot 08$ & $0.81,1.44$ \\
\hline Mid-morning snackll & $1 \cdot 15$ & $0.79,1.67$ & $0 \cdot 86$ & $0.57,1.30$ & $1 \cdot 77$ & $1 \cdot 22,2 \cdot 57$ & $1 \cdot 21$ & $0 \cdot 80,1 \cdot 81$ \\
\hline Afternoon snackll & 1.60 & $1 \cdot 26,2 \cdot 02$ & $1 \cdot 43$ & $1 \cdot 11,1 \cdot 84$ & $1 \cdot 78$ & $1 \cdot 40,2 \cdot 27$ & $1 \cdot 34$ & $1 \cdot 03,1 \cdot 75$ \\
\hline Besides meals snackll & 1.51 & $1 \cdot 21,1 \cdot 87$ & $1 \cdot 41$ & $1 \cdot 12,1 \cdot 77$ & 1.59 & $1.27,1.99$ & $1 \cdot 46$ & $1 \cdot 14,1 \cdot 86$ \\
\hline Monitoring weight & $1 \cdot 16$ & $0.94,1.44$ & $1 \cdot 07$ & $0 \cdot 86,1 \cdot 35$ & $1 \cdot 19$ & $0.93,1.52$ & $1 \cdot 10$ & $0 \cdot 85,1 \cdot 44$ \\
\hline Dieting & $5 \cdot 75$ & $3.97,8 \cdot 35$ & $4 \cdot 60$ & $3 \cdot 12,6 \cdot 78$ & 3.94 & $2 \cdot 98,5 \cdot 20$ & $2 \cdot 75$ & $2 \cdot 03,3 \cdot 71$ \\
\hline $15-18$ years old & \multicolumn{2}{|c|}{$(n 2196)$} & \multicolumn{2}{|c|}{ (n 2196) } & \multicolumn{2}{|c|}{$(n$ 3663) } & \multicolumn{2}{|c|}{$(n$ 3663) } \\
\hline Physical activity & & & & & & & & \\
\hline Up to $7 \mathrm{~h} /$ week & 1.00 & Ref. & 1.00 & Ref. & 1.00 & Ref. & 1.00 & Ref. \\
\hline$>7 \mathrm{~h} /$ week & 0.97 & $0.73,1.27$ & 1.08 & $0.80,1.46$ & $1 \cdot 04$ & $0.79,1.35$ & $1 \cdot 06$ & $0 \cdot 80,1 \cdot 40$ \\
\hline \multicolumn{9}{|l|}{ TV watching } \\
\hline Up to $7 \mathrm{~h} /$ week & 1.00 & Ref. & 1.00 & Ref. & 1.00 & Ref. & 1.00 & Ref. \\
\hline$>7 \mathrm{~h} /$ week & 0.73 & $0.55,0.95$ & 0.69 & $0.51,0.92$ & $1 \cdot 31$ & $1 \cdot 01,1 \cdot 70$ & $1 \cdot 34$ & $1 \cdot 02,1 \cdot 76$ \\
\hline \multicolumn{9}{|l|}{ PC use } \\
\hline Up to $3 \mathrm{~h} /$ week & $1 \cdot 00$ & Ref. & 1.00 & Ref. & 1.00 & Ref. & 1.00 & Ref. \\
\hline$>3 \mathrm{~h} /$ week & 0.96 & $0 \cdot 71,1 \cdot 29$ & $1 \cdot 04$ & $0 \cdot 76,1 \cdot 42$ & $0 \cdot 87$ & $0 \cdot 64,1 \cdot 18$ & 0.79 & $0 \cdot 57,1 \cdot 10$ \\
\hline Breakfastll & $1 \cdot 41$ & $1.05,1.90$ & $1 \cdot 31$ & $0.95,1.79$ & $1 \cdot 26$ & $0.98,1.63$ & $1 \cdot 17$ & $0.89,1.53$ \\
\hline Lunchll & $1 \cdot 74$ & $1 \cdot 28,2 \cdot 37$ & 1.65 & $1 \cdot 18,2 \cdot 30$ & $1 \cdot 85$ & $1 \cdot 42,2 \cdot 41$ & $1 \cdot 49$ & $1.13,1.97$ \\
\hline Mid-morning snackll & $2 \cdot 05$ & $1 \cdot 48,2 \cdot 86$ & $1 \cdot 76$ & $1 \cdot 25,2 \cdot 50$ & $1 \cdot 20$ & $0 \cdot 88,1 \cdot 65$ & $1 \cdot 15$ & $0.82,1 \cdot 61$ \\
\hline Afternoon snackll & 1.91 & $1 \cdot 43,2.55$ & 1.56 & $1 \cdot 14,2 \cdot 15$ & 1.51 & $1 \cdot 16,1 \cdot 98$ & $1 \cdot 29$ & $0.97,1.71$ \\
\hline Besides meals snackll & 1.97 & $1 \cdot 50,2 \cdot 60$ & $1 \cdot 71$ & $1 \cdot 27,2 \cdot 28$ & 1.47 & $1 \cdot 14,1 \cdot 90$ & $1 \cdot 31$ & $0.99,1.71$ \\
\hline Monitoring weight & $1 \cdot 25$ & $0.95,1.65$ & $1 \cdot 17$ & $0.87,1.57$ & 0.89 & $0.68,1 \cdot 17$ & 0.68 & $0.51,0.92$ \\
\hline Dieting & $2 \cdot 87$ & $1 \cdot 40,5 \cdot 89$ & $2 \cdot 05$ & $0.93,4.48$ & $3 \cdot 11$ & $2 \cdot 33,4 \cdot 15$ & $2 \cdot 87$ & $2.09,3.95$ \\
\hline
\end{tabular}

IOTF, International Obesity Task Force; Ref., reference category. Bold values are significantly different from $1(P<0.05)$.

$\ddagger$ Crude model with each behavioural factor analysed separately on BMI, adjusted for school and age.

§Adjusted model with all behavioural factors included in analyses on BMI, adjusted for school, age, birth weight, breast-feeding, puberty, parents' BMI, parents' education, number of inhabitants and family size.

II'Yes' is reference category $v$. 'no'

' 'No' is reference category $v$. 'yes'.

Among girls, dieting (10-14-year-olds: OR $=2 \cdot 75,95 \%$ CI $2 \cdot 03,3 \cdot 71 ; 15-18$-year-olds: OR $=2 \cdot 87,95 \%$ CI $2 \cdot 09$, 3.95) was positively associated with being overweight/ obese in both age groups, with slightly higher odds among the older girls. The 10-14-year-old girls further showed positive associations on being overweight/obese when skipping an afternoon snack $(\mathrm{OR}=1 \cdot 34,95 \% \mathrm{CI}$ $1 \cdot 03,1 \cdot 75)$ and when not having a besides meals snack $(\mathrm{OR}=1 \cdot 46,95 \% \mathrm{CI} 1 \cdot 14,1 \cdot 86)$. These relations were not found in 15-18-year-old girls. However, among these girls, skipping lunch $(\mathrm{OR}=1.49,95 \% \mathrm{CI} 1 \cdot 13,1.97)$ and watching TV for $>7 \mathrm{~h} /$ week $(\mathrm{OR}=1 \cdot 34,95 \% \mathrm{CI} 1 \cdot 02,1 \cdot 76)$ were positively associated with being overweight/obese. Negative associations were also found in 15-18-year-old girls for monitoring weight ( $\mathrm{OR}=0 \cdot 68,95 \% \mathrm{CI} 0 \cdot 51,0 \cdot 92)$ and being overweight/obese.
When comparing the 'youngest' boys and girls, not having an afternoon snack or a besides meal snack and dieting were positively associated with being overweight/ obese between both genders. It was only among boys that inverse associations with being overweight/obese were found when being highly physically active for $>7 \mathrm{~h} /$ week. In addition, skipping breakfast was also found to be positively associated with being overweight/ obese in boys.

Among the 'oldest' groups, skipping lunch was found to be positively associated with being overweight/obese between both genders. Boys further showed positive associations with overweight/obesity when skipping other meals like mid-morning snack, afternoon snack and besides meals snack. It was only among girls that dieting and watching TV for $>7 \mathrm{~h} /$ week were positively associated 
with being overweight/obese. For boys, negative associations with a high amount of TV viewing $(>7 \mathrm{~h} /$ week) on being overweight/obese were found. Negative associations on overweight/obesity were also found among girls with monitoring of their weight.

In addition to the multi-level logistic regression models, multinomial regression models were run to be able to compare the three weight groups. Comparable results were found for overweight boys and girls. Higher risks for being 'obese' were found in boys and girls when they reported being on a diet and when they skipped a besides meals snack. Among boys, skipping an afternoon snack was also positively associated with being obese (data not shown).

\section{Discussion}

The aim of the present study was to assess prevalence of normal weight, overweight or being obese among 10-18-year-old Czech Republic adolescents and to research which associations were being found between energy balance-related behaviours and overweight/obesity among these adolescents.

Czech Republic adolescent boys were shown to have a somewhat higher prevalence of overweight and obesity than girls, independent of age. Prevalence of overweight varied between $6 \cdot 1 \%$ and $16 \cdot 7 \%$ and that for obesity between $0.7 \%$ and $3.7 \%$. Other studies found similar results among European adolescents within the same age range ${ }^{(12)}$.

Most of the behavioural factors were independently statistically significantly associated with being overweight/obese. This mostly changed when adding all behavioural factors and background factors together in the model and suggested an overlap among factors, although intercorrelations were below $0 \cdot 50$.

Positive associations of dieting, high amount of watching TV and skipping meals on being overweight/ obese, and negative associations of high physical activity and monitoring weight on being overweight/obese were found in the present study. Similar results were found in other studies and reviews ${ }^{(6,13-19)}$. With regard to dieting and skipping meals, contradictory results have been found in the literature. The presence of reverse causality has been indicated, with dieting and skipping meals as the consequences of being overweight/obese ${ }^{(15)}$, while other research suggests a causal relationship between skipping a meal and becoming overweight or obese. Under-reporting of daily consumption of meals might also be a factor of the positive association between skipping meals and overweight/obesity ${ }^{(6)}$. Since the present data are cross-sectional, the direction of the relationship regarding dieting and skipping meals cannot be estimated.

The effect of school as a clustering variable was minor in the present study. Yet intervention studies and randomized controlled trials on behaviours for preventing and treating overweight and obesity have shown a school effect ${ }^{(20,21)}$, which suggests that future prevention strategies might include school policy and neighbourhood components ${ }^{(22,23)}$.

A surprising finding in our study was an inverse association of high amounts of watching TV with being overweight/obese in older boys. This is in contrast with findings from the literature. A possible explanation could be that under-reporting in overweight/obese boys was present, which led to statistically significant negative associations in the non-overweight/obese boys.

\section{Strengths and limitations}

The present study has some strengths and limitations. The strength of the present study is that BMI was calculated with objectively measured height and weight. Objectively measured height and weight, instead of self-reported height and weight, are more accurate since self-reporting, especially in children and adolescents, could lead to biased results. Another strength is the use of internationally accepted gender- and age-specific cut-off values $^{(7)}$, which enables comparison with similar studies performed in other countries.

The large sample size can also be considered a strength of the present study. It implicates that precautions should be taken when interpreting results from the analyses. Statistical significance is highly likely in the present study. We therefore chose to use a $P$ value of $0 \cdot 01$ when assessing associations with energy balance-related behaviours and background factors on normal weight, overweight and being obese. Moreover, when performing multi-level logistic regression analysis, the total sample was clustered for school and divided into four groups based on age and gender, resulting in moderate sample sizes. Possible hierarchical structures in the data have been taken into account by performing multi-level modelling. In addition, the analysis in four groups takes possible different patterns in behaviours between age and gender into account, which are shown in the results of the present study. Moreover, multinomial regression analyses, which were performed in addition to assess associations of behaviours with being overweight or obese, showed similar findings.

A large number of schools from all over the country were included. We therefore believe that this sample is representative, although dropout analysis revealed that overweight and obese adolescents dropped out more often.

A weakness of the present study is that the parents' and children's questionnaires were not pre-tested, resulting in lack of information on validity and reliability. Further, behavioural and background variables consisted of selfreported single-item questions. This could have led to under- or over-reporting or interpretation inaccuracies between the different ages.

For analysing the possible associations between behavioural factors and BMI, cross-sectional data from the Czech Republic has been used. Associations between the 
behaviours and being overweight/obese should therefore be carefully interpreted.

The present data were collected in 2001. The prevalence of overweight and obesity should be monitored more often to observe and measure the effectiveness of national prevention strategies.

The results of the present study provide information on the prevalence and associations of energy balance-related behaviours on overweight and obesity in a country where not much information on this topic is as yet present. Moreover, finding literature on intervention studies for preventing overweight and obesity, performed in this country, was difficult. Therefore, results from the present study are important for emphasizing the obesity problem and the need for executing (intervention) studies on overweight and obesity in the Czech Republic.

To conclude, the present study revealed that skipping a meal, independent of the main meal or snack and dieting are associated with being overweight or obese in Czech Republic adolescents, independent of gender. In the future, the results of the present study can be useful when performing overweight and obesity prevention programmes in the Czech Republic. However, more preferably, longitudinal, national studies using better behavioural measures are needed. Additional measures combining a multi-level approach within the adolescents' environment should be investigated for making future interventions successful.

\section{Acknowledgements}

Sources of funding: The present paper has been facilitated by the EU-funded HOPE project: Health Promotion through Obesity Prevention across Europe (the Commission of the European Communities, SP5A-CT-2006-044128). The study does not necessarily reflect the Commission's views and in no way anticipates the Commission's future policy in this area. The data collection was supported by the Internal Grant Agency of MH, Czech Republic, Grant no. NJ/6792-3 (The 6th Nation-wide Anthropological Survey of Children and Adolescents 2001, Czech Republic). Conflicts of interest declaration: There are no conflicts of interest to be declared. Author contributions: The authors participated in the following ways: L.d.G., N.L. and M.W. conceptualized the study questions. J.V. provided the data on adolescents in the Czech Republic. L.d.G. conducted the analyses and prepared the manuscript with the help of M.W., K.I.K., N.L., I.S. and M.W. provided statistical expertise. All co-authors made contributions in refining the manuscript drafts. M.W. provided overall guidance in data analysis and interpretation and writing of the manuscript.

\section{References}

1. Humenikova L \& Gates GE (2007) Dietary intakes, physical activity, and predictors of child obesity among 4-6th graders in the Czech Republic. Cent Eur J Publ Health 15, 23-28.
2. Parízkóva J (2000) Dietary habits and nutritional status in adolescents in Central and Eastern Europe. Eur J Clin Nutr 54, Suppl. 1, S36-S40.

3. World Health Organization (2002) The World Health Report. Geneva: WHO; available at http://www.who.int/entity/ whr/2003/en/whr03_en.pdf

4. Ebbeling CB, Pawlak DB \& Ludwig DS (2002) Childhood obesity: public health crisis, common sense cure. Lancet 10, 473-482.

5. Prentice AM (2006) The emerging epidemic of obesity in developing countries. Int J Epidemiol 35, 93-99.

6. Rennie KL, Johnson L \& Jebb SA (2005) Behavioural determinants of obesity. Best Pract Res Clin Endocrinol Metab 19, 343-358.

7. Cole TJ, Bellizzi MC, Flegal KM et al. (2000) Establishing a standard definition for child overweight and obesity: international survey. BMJ 320, 1240-1243.

8. Kobzová J, Vignerová J, Bláha P et al. (2004) The 6th nationwide anthropological survey of children and adolescents in the Czech Republic in 2001. Cent Eur J Public Health 12, 126-130.

9. Miles JNV \& Shevlin ME (2001) Applying Regression and Correlation: A Guide for Students and Researchers. London: Sage Publications.

10. Toschke A, Vignerová J, Lbotska L et al. (2002) Overweight and obesity in 6- to 14-year-old Czech children in 1991: protective effect of breast-feeding. J Pediatr 141, 764-769.

11. Rasbash J, Charlton C, Browne WJ, et al. (2009) MLwiN Version 2.1. Centre for Multilevel Modelling, University of Bristol.

12. Janssen I, Katzmarzyk PT, Boyce WF et al. (2005) Comparison of overweight and obesity prevalence in school-aged youth from 34 countries and their relationships with physical activity and dietary patterns. Obes Rev 6, 123-132.

13. Sweeting HN (2008) Gendered dimensions of obesity in childhood and adolescence. Nutr J7.1.

14. Levitsky DA, Garay J, Nausbaum M et al. (2006) Monitoring weight daily blocks the freshman weight gain: a model for combating the epidemic of obesity. Int J Obes 30, 1003-1010.

15. Newby PK (2007) Are dietary intakes and eating behaviors related to childhood obesity? A comprehensive review of the evidence. J Law Med Ethics 35, 35-60.

16. Sherry B (2005) Food behaviors and other strategies to prevent and treat pediatric overweight. Int J Obes 29, S116-S126.

17. Spear BA, Barlow SE, Ervin C et al. (2007) Recommendations for treatment of child and adolescent overweight and obesity. Pediatrics 120, S254-S288.

18. Wareham NJ, Sluijs EMF \& Ekelund U (2005) Physical activity and obesity prevention: a review of the current evidence. Proc Nutr Soc 64, 229-247.

19. Zaborskis A, Petronyte G, Sumskas L et al. (2008) Body image and weight control among adolescents in Lithuania, Croatia, and the United States in the context of global obesity. Croat Med J 49, 233-242.

20. Oude Luttikhuis H, Baur L, Jansen H et al. (2009) Interventions for treating obesity in children. Cochrane Database Syst Rev, issue 1, Art. No.: CD001872.

21. Veugelers PJ \& Fitzgerald AL (2005) Effectiveness of school programs in preventing childhood obesity: a multilevel comparison. Am J Public Health 95, 432-435.

22. Harrington DW \& Elliot SJ (2009) Weighing the importance of neighbourhood: a multilevel exploration of the determinants of overweight and obesity. Soc Sci Med 68, 593-600.

23. Kumanyika SK, Obarzanek E, Stettler N et al. (2008) Population-based prevention of obesity the need for comprehensive promotion of healthful eating, physical activity, and energy balance a scientific statement from American Heart Association Council on epidemiology and prevention, interdisciplinary committee for prevention (formerly the expert panel on population and prevention science). Circulation 118, 428-464. 